\title{
THE NORM MAP AND THE CAPITULATION KERNEL
}

\author{
CRISTIAN D. GONZÁLEZ-AVILÉS
}

\begin{abstract}
Let $f: S^{\prime} \rightarrow S$ be a finite and faithfully flat morphism of locally noetherian schemes of constant rank $n \geq 2$ and let $G$ be a smooth, commutative and quasi-projective $S$-group scheme with connected fibers. Under certain restrictions on $f$ and $G$, we relate the kernel of the restriction map $\operatorname{Res}_{G}^{(r+1)}: H^{r+1}\left(S_{\text {ét }}, G\right) \rightarrow H^{r+1}\left(S_{\text {ét }}^{\prime}, G\right)$ in étale cohomology, where $r \geq 0$, to a quotient of the kernel of the $\bmod n$ corestriction map $\operatorname{Cores}_{G}^{(r)} / n: H^{r}\left(S_{\text {ét }}^{\prime}, G\right) / n \rightarrow H^{r}\left(S_{\text {ét }}, G\right) / n$. When $r=0$ and $f$ is a Galois covering with Galois group $\Delta$, our main theorem relates $\operatorname{Ker} \operatorname{Res}_{G}^{(1)}=H^{1}\left(\Delta, G\left(S^{\prime}\right)\right)$ to the subgroup of $G\left(S^{\prime}\right)$ of sections whose $\left(S^{\prime} / S\right)$-norm lies in $G(S)^{n}$. Applications are given to the capitulation problem for Néron-Raynaud class groups of tori and Tate-Shafarevich groups of abelian varieties.
\end{abstract}

\section{INTRODUCTION}

Let $f: S^{\prime} \rightarrow S$ be a finite and faithfully flat morphism of locally noetherian schemes of constant rank $n \geq 2$ and let $G$ be a smooth, commutative and quasi-projective $S$-group scheme with connected fibers. In this paper we re-

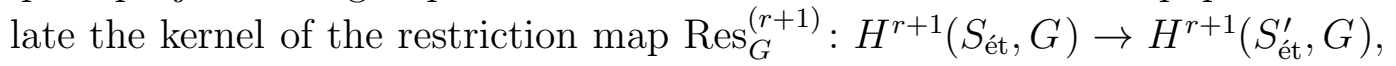
where $r \geq 0$, to a certain quotient of the kernel of the mod $n$ corestriction (or norm) map $\operatorname{Cores}_{G}^{(r)} / n: H^{r}\left(S_{\text {ét }}^{\prime}, G\right) / n \rightarrow H^{r}\left(S_{\text {ét }}, G\right) / n$ when $(f, G)$ is admissible, which means that the following conditions hold at every point $s \in S$ such that char $k(s)$ divides $n:\left(\right.$ i) $f_{s}: S^{\prime} \times{ }_{S} \operatorname{Spec} k(s) \rightarrow \operatorname{Spec} k(s)$ is étale (i.e., unramified), and (ii) $G_{s}$ is a semiabelian $k(s)$-variety. For example, $(f, G)$ is admissible if $f$ is étale and $G$ is a semiabelian $S$-scheme. See below for more examples. In order to simplify the exposition in this Introduction, we

2010 Mathematics Subject Classification. Primary 11R29, 14F20.

Key words and phrases. Capitulation kernel, relative Brauer group, Tate-Shafarevich group, Néron-Raynaud class group, ideal class group, quadratic Galois covers.

The author was partially supported by Fondecyt grant 1160004. 
state below a corollary of our main theorem (Theorem 4.2) in the following case: $r=0, f$ is a Galois covering with Galois group $\Delta$ (in particular, $f$ is étale) and $G=\mathbb{G}_{m, S}$ is the multiplicative group scheme over $S$. In this case Ker $\operatorname{Res}_{G}^{(1)} \simeq \operatorname{KerPic} f \simeq H^{1}\left(\Delta, U\left(S^{\prime}\right)\right)$, where Pic $f: \operatorname{Pic} S \rightarrow \operatorname{Pic} S^{\prime}$ is the canonical map induced by $f$ and $U\left(S^{\prime}\right)=\Gamma\left(S^{\prime}, \mathcal{O}_{S^{\prime}}\right)^{*}$ is the group of global units on $S^{\prime 1}$. Consider the following abelian fppf sheaf on $S$ :

$$
\mathbb{G}_{m}(n)=R_{S^{\prime} / S}^{(1)}\left(\mu_{n}\right) / \mu_{n, S},
$$

where $R_{S^{\prime} / S}^{(1)}\left(\mu_{n}\right)=\operatorname{Ker}\left[N_{S^{\prime} / S}: R_{S^{\prime} / S}\left(\mu_{n, S^{\prime}}\right) \rightarrow \mu_{n, S}\right]$ is the $S$-group scheme of $n$-th roots of unity on $S^{\prime}$ of $\left(S^{\prime} / S\right)$-norm 1 . The sheaf (1.1) is an $\left(S^{\prime} / S\right)$-form of $\mu_{n, S}^{n-2}$, i.e., $\mathbb{G}_{m}(n)_{S^{\prime}} \simeq \mu_{n, S^{\prime}}^{n-2}$. Now set

$$
\Psi_{N}(n, U)=\left\{x \in U\left(S^{\prime}\right): N_{S^{\prime} / S}(x) \in U(S)^{n}\right\} .
$$

Then the following holds

Theorem 1.1. There exists a canonical exact sequence of $n$-torsion abelian groups

$$
\begin{aligned}
1 & \rightarrow U(S) \cap U\left(S^{\prime}\right)^{n} / U(S)^{n} \rightarrow H^{1}\left(\Delta, \mu_{n}\left(S^{\prime}\right)\right) \rightarrow \text { Ker Pic } f \\
& \rightarrow \Psi_{N}(n, U) / U(S) U\left(S^{\prime}\right)^{n} \rightarrow H^{1}\left(\Gamma_{n}^{\bullet}(U)\right),
\end{aligned}
$$

where $\Psi_{N}(n, U)$ is the group (1.2) and

$$
H^{1}\left(\Gamma_{n}^{\bullet}(U)\right)=\frac{\operatorname{Ker}\left[H^{1}\left(S_{\mathrm{fl}}, \mathbb{G}_{m}(n)\right) \rightarrow H^{2}\left(S_{\mathrm{fl}}, \mu_{n}\right)\right]}{\operatorname{Im}\left[H^{0}\left(S_{\mathrm{fl}}, \mu_{n}\right) \rightarrow H^{1}\left(S_{\mathrm{fl}}, \mathbb{G}_{m}(n)\right)\right]},
$$

where $\mathbb{G}_{m}(n)$ is the sheaf (1.1).

If $\Delta$ is cyclic, then the above theorem is neither surprising nor optimal since the group

$$
\text { Ker Pic } f \simeq H^{1}\left(\Delta, U\left(S^{\prime}\right)\right) \simeq H^{-1}\left(\Delta, U\left(S^{\prime}\right)\right) \quad \text { (Tate cohomology) }
$$

is a quotient of $\left\{x \in U\left(S^{\prime}\right): N_{S^{\prime} / S}(x)=1\right\}$, whence Ker Pic $f$ can be directly related to a subgroup of $U\left(S^{\prime}\right)$ defined in terms of the norm map $N_{S^{\prime} / S}$. Note, however, that the theorem shows (in particular) that Ker Pic $f$ can be related to such a subgroup of $U\left(S^{\prime}\right)$ for any $\Delta$.

\footnotetext{
${ }^{1}$ The isomorphism Ker Pic $f \simeq H^{1}\left(\Delta, U\left(S^{\prime}\right)\right)$ follows from the Hochschild-Serre spectral sequence associated to the Galois covering $S^{\prime} / S$.
} 
Another class of admissible pairs is the following. Let $F$ be a number field, write $\mathcal{O}_{F}$ for the ring of integers of $F$ and let $K / F$ be a finite Galois extension of degree $n$ which is unramified over the set $\Omega$ of primes of $F$ that divide $n$. Let $G_{F}$ be either an abelian variety or an algebraic torus over $F$ with semiabelian reduction over $\Omega$ 2. If $f: \operatorname{Spec} \mathcal{O}_{K} \rightarrow \operatorname{Spec} \mathcal{O}_{F}$ is the morphism induced by the inclusion $\mathcal{O}_{F} \subset \mathcal{O}_{K}$ and $G$ denotes the identity component of the Néron-Raynaud model of $G_{F}$ over $\mathcal{O}_{F}$, then $(f, G)$ is an admissible pair. In this setting our main Theorem 4.2 yields information on the Capitulation Problem for the Tate-Shafarevich group of $G_{F}$ over $F$ and the Néron-Raynaud class group of $G_{F}$ introduced in [11, §3]. See Section [6] for the details.

The paper is organized as follows. Section 2 consists of preliminaries, in particular on the Weil restriction functor. In section 3 we discuss the norm one group scheme, which plays a central role in the paper. In section 4 we establish our main theorem (Theorem 4.2). The developments of this section were inspired by [23, §3] but, in contrast to [loc.cit.], we avoid working with hypercohomological spectral sequences and work instead primarily with complexes of length 3. In section 5 we specialize our results to the (interesting) case of quadratic Galois coverings. Section [6, which concludes the paper, discusses the applications of our main theorem to the Capitulation Problem mentioned above for quadratic Galois extensions of global fields.

\section{ACKNOWLEDGEMENTS}

I thank Laurent Moret-Bailly for answering my question [21]. I also thank the referee for pointing out the need to include additional applications of the main theorem, which motivated the inclusion of Section 6 in this version. This research was partially supported by Fondecyt grant 1160004 .

\section{Preliminaries}

If $n \geq 1$ is an integer and $A$ is an object of an abelian category $\mathscr{A}, A_{n}$ (respectively, $A / n$ ) will denote the kernel (respectively, cokernel) of the multiplication by $n$ morphism on $A$. If $\psi: A \rightarrow B$ is a morphism in $\mathscr{A}, \psi_{n}: A_{n} \rightarrow B_{n}$ and $\psi / n: A / n \rightarrow B / n$ will denote the morphisms in $\mathscr{A}$ induced by $\psi$. Note that

$$
\operatorname{Ker}\left(\psi_{n}\right)=(\operatorname{Ker} \psi)_{n} \quad \text { and } \quad \operatorname{Coker}(\psi / n)=(\operatorname{Coker} \psi) / n \text {. }
$$

\footnotetext{
${ }^{2}$ Thus, if $G_{F}$ is an $F$-torus, then $G_{F}$ has multiplicative (i.e., toric) reduction over $\Omega$.
} 
Proposition 2.1. Let $A \stackrel{f}{\rightarrow} B \stackrel{g}{\rightarrow} C$ be morphisms in an abelian category $\mathscr{A}$. Then there exists a canonical exact sequence in $\mathscr{A}$

$0 \rightarrow \operatorname{Ker} f \rightarrow \operatorname{Ker}(g \circ f) \rightarrow \operatorname{Ker} g \rightarrow$ Coker $f \rightarrow \operatorname{Coker}(g \circ f) \rightarrow$ Coker $g \rightarrow 0$.

Proof. See, for example, [4, 1.2]. The middle map Ker $g \rightarrow$ Coker $f$ is the composition Ker $g \hookrightarrow B \rightarrow$ Coker $f$. The remaining maps are the natural ones.

All schemes below are tacitly assumed to be non-empty.

If $S$ is a scheme and $\tau$ (= ét or fl) denotes either the étale or the fppf topology on $S, S_{\tau}$ will denote the small $\tau$ site over $S$. A faithfully flat morphism locally of finite presentation $S^{\prime} \rightarrow S$ is an fppf covering of $S$. We will write $S_{\tau}^{\sim}$ for the category of sheaves of abelian groups on $S_{\tau}$, which is abelian. If $G$ is a commutative $S$-group scheme, the presheaf represented by $G$ is an object of $S_{\tau}^{\sim}$. In particular, if $f: S^{\prime} \rightarrow S$ is an fppf covering of $S$ as above, then the map $G(S) \hookrightarrow G\left(S^{\prime}\right)$ induced by $f$ is an injection that will be regarded as an inclusion. If $n \geq 1$ is an integer, the object $G_{n}$ of the abelian category $S_{\tau}^{\sim}$ is represented by the $S$-group scheme $G \times_{n_{G}, G, \varepsilon} S$, where $n_{G}$ is the $n$-th power morphism on $G$ and $\varepsilon: S \rightarrow G$ is the unit section of $G$. We will make the identifications

$$
n_{G_{S^{\prime}}}=n_{G} \times{ }_{S} S^{\prime} \quad \text { and } \quad G_{n, S^{\prime}}=\left(G_{n}\right)_{S^{\prime}}=\left(G_{S^{\prime}}\right)_{n} .
$$

If $G$ is separated over $S$, then $G_{n} \hookrightarrow G$ is a closed immersion. If, in addition, $G$ is quasi-projective over $S$, then $G_{n}$ is also quasi-projective over $S$ [14, II, Proposition 5.3.4(i)]. If $\psi: G \rightarrow H$ is a morphism of commutative $S$-group schemes, $\psi_{n}: G_{n} \rightarrow H_{n}$ will denote the morphism of $S$-group schemes induced by $\psi[15,(1.2 .3)$, pp. 26-27].

If $G$ is as above, we will write $H^{r}\left(S_{\tau}^{\prime}, G\right)$ for $H^{r}\left(S_{\tau}^{\prime}, G_{S^{\prime}}\right)$, where $G_{S^{\prime}}=$ $G \times{ }_{S} S^{\prime}$. If $G$ is smooth over $S, H^{r}\left(S_{\mathrm{fl}}, G\right)$ and $H^{r}\left(S_{\text {ét }}, G\right)$ will be identified via [17, Theorem 11.7(1), p. 180]. If $G=\mathbb{G}_{m, S}$, the groups $H^{r}\left(S_{\tau}, G\right)$ will be denoted by $H^{r}\left(S_{\tau}, \mathbb{G}_{m}\right)$. Further, we will identify $H^{1}\left(S_{\text {ét }}, \mathbb{G}_{m}\right)$ and Pic $S$ via [20, Theorem 4.9, p. 124] and we will write $\operatorname{Br}^{\prime} S$ for the cohomological Brauer group of $S$, i.e., $\operatorname{Br}^{\prime} S=H^{2}\left(S_{\text {ét }}, \mathbb{G}_{m}\right)$. Let $\operatorname{Br} S$ denote the Brauer group of equivalence classes of Azumaya algebras on $S$. If $S$ is quasi-compact and admits an ample invertible sheaf, then the canonical map $\operatorname{Br} S \rightarrow \mathrm{Br}^{\prime} S$ 
induces an isomorphism of torsion abelian groups

$$
\operatorname{Br} S \simeq\left(\mathrm{Br}^{\prime} S\right)_{\text {tors }}
$$

by [18] and [25, Tag 01PR, Lemma 27.26.8].

A sequence of commutative $S$-group schemes

$$
0 \rightarrow G_{1} \rightarrow G_{2} \rightarrow G_{3} \rightarrow 0
$$

will be called exact if the corresponding sequence of representable objects in $S_{\mathrm{fl}}^{\sim}$ is exact. If $G_{2} \rightarrow G_{3}$ is faithfully flat and locally of finite presentation and $G_{1}=G_{2} \times_{G_{3}} S$ denotes the scheme-theoretic kernel of $G_{2} \rightarrow G_{3}$, then (2.3) is exact.

Let $f: S^{\prime} \rightarrow S$ be a finite and faithfully flat morphism of locally noetherian schemes of constant rank $n \geq 2$ and let $X^{\prime}$ be an $S^{\prime}$-scheme. The Weil restriction of $X^{\prime}$ along $f$ is the contravariant functor $(\mathrm{Sch} / S) \rightarrow$ (Sets) defined by $T \mapsto \operatorname{Hom}_{S^{\prime}}\left(T \times_{S} S^{\prime}, X^{\prime}\right)$. This functor is representable if there exist an $S$-scheme $R_{S^{\prime} / S}\left(X^{\prime}\right)$ and a morphism of $S^{\prime}$-schemes

$$
\theta_{X^{\prime}, S^{\prime} / S}: R_{S^{\prime} / S}\left(X^{\prime}\right)_{S^{\prime}} \rightarrow X^{\prime}
$$

such that the map

$$
\operatorname{Hom}_{S}\left(T, R_{S^{\prime} / S}\left(X^{\prime}\right)\right) \rightarrow \operatorname{Hom}_{S^{\prime}}\left(T \times_{S} S^{\prime}, X^{\prime}\right), \quad g \mapsto \theta_{X^{\prime}, S^{\prime} / S} \circ g_{S^{\prime}},
$$

is a bijection (functorially in $T$ ). See [6, $\$ 7.6]$ and [7, Appendix A.5] for basic information on the Weil restriction functor 3 . The map $\theta_{X^{\prime}, S^{\prime} / S}$ (2.4) is both functorial in $X^{\prime}$ and compatible with compositions and arbitrary base changes [5. (2.40) and (2.41), p. 16]. We will write

$$
j_{X, S^{\prime} / S}: X \rightarrow R_{S^{\prime} / S}\left(X_{S^{\prime}}\right)
$$

for the canonical adjunction $S$-morphism, i.e., the $S$-morphism that corresponds to the identity morphism of $X_{S^{\prime}}$ under the bijection (2.5).

For lack of an adequate reference, we include here a proof of

\footnotetext{
${ }^{3}$ As noted by Brian Conrad, the restriction in [7, Appendix A.5] to an affine base $S$ can be removed since all assertions in [loc.cit.] are local on $S$. Further, the noetherian hypotheses in [loc.cit.] are satisfied since we are assuming that $S$ and $S^{\prime}$ are locally noetherian. Finally, we note that the quasi-projectivity hypotheses in [loc.cit.] are only needed to guarantee the existence of the relevant Weil restrictions in the category of schemes.
} 
Proposition 2.2. Let $n \geq 1$ be an integer and let $S_{i}^{\prime} \rightarrow S$ (where $\left.1 \leq i \leq n\right)$ be a finite collection of $\bar{S}$-schemes. Set $S^{\prime}=\coprod_{i=1}^{n} S_{i}^{\prime}$ and let $S^{\prime} \rightarrow \bar{S}$ be the canonical morphism of schemes induced by the given morphisms $S_{i}^{\prime} \rightarrow S$. Then, for every $S^{\prime}$-scheme $X^{\prime}$, there exists an isomorphism of functors

$$
R_{S^{\prime} / S}\left(X^{\prime}\right) \stackrel{\sim}{\rightarrow} \prod_{i=1}^{n} R_{S_{i}^{\prime} / S}\left(X^{\prime} \times_{S^{\prime}} S_{i}^{\prime}\right) .
$$

Proof. Let $Y$ be any $S$-scheme. Since the tensor product is distributive with respect to finite direct products, (2.5) and [15, §3.1, pp. 230-231] yield canonical bijections

$$
\begin{aligned}
\operatorname{Hom}_{S}\left(Y, R_{S^{\prime} S}\left(X^{\prime}\right)\right) & \stackrel{\sim}{\rightarrow} \operatorname{Hom}_{S^{\prime}}\left(Y \times_{S} S^{\prime}, X^{\prime}\right) \\
& \simeq \operatorname{Hom}_{S^{\prime}}\left(\coprod_{i=1}^{n}\left(Y \times_{S} S_{i}^{\prime}\right), X^{\prime}\right) \\
& \simeq \prod_{i=1}^{n} \operatorname{Hom}_{S^{\prime}}\left(Y \times_{S} S_{i}^{\prime}, X^{\prime}\right) \\
& \simeq \prod_{i=1}^{n} \operatorname{Hom}_{S_{i}^{\prime}}\left(Y \times_{S} S_{i}^{\prime}, X^{\prime} \times_{S} S_{i}^{\prime}\right) \\
& \simeq \prod_{i=1}^{n} \operatorname{Hom}_{S}\left(Y, R_{S_{i}^{\prime} / S}\left(X^{\prime} \times_{S^{\prime}} S_{i}^{\prime}\right)\right) \\
& \simeq \operatorname{Hom}_{S}\left(Y, \prod_{i=1}^{n} R_{S_{i}^{\prime} / S}\left(X^{\prime} \times_{S^{\prime}} S_{i}^{\prime}\right)\right) .
\end{aligned}
$$

The proposition follows.

If $n \geq 1$ is an integer and $X$ is an $S$-scheme, $X^{n}$ will denote the $S$-scheme defined recursively by $X^{1}=X$ and $X^{n}=X \times_{S} X^{n-1}$ for $n \geq 2$.

Corollary 2.3. Let $X$ be an $S$-scheme such that the $S$-scheme $R_{S^{\prime} / S}\left(X_{S^{\prime}}\right)$ exists and let $S^{\prime \prime} \rightarrow S$ be a morphism of schemes such that $S^{\prime} \times{ }_{S} S^{\prime \prime} \simeq \coprod_{i=1}^{n} S^{\prime \prime}$, where $n \geq 1$. Then there exists a canonical isomorphism of $S^{\prime \prime}$-schemes

$$
R_{S^{\prime} / S}\left(X_{S^{\prime}}\right) \times_{S} S^{\prime \prime} \simeq X_{S^{\prime \prime}}^{n}
$$

Proof. This is immediate from the proposition via the canonical isomorphism of $S^{\prime \prime}$-schemes $R_{S^{\prime} / S}\left(X_{S^{\prime}}\right) \times_{S} S^{\prime \prime} \simeq R_{S^{\prime} \times_{S} S^{\prime \prime} / S^{\prime \prime}}\left(X_{S^{\prime} \times{ }_{S} S^{\prime \prime}}\right)$.

\section{THE NORM ONE GROUP SCHEME}

Let $f: S^{\prime} \rightarrow S$ be a finite and faithfully flat morphism of locally noetherian schemes of constant rank $n \geq 2$ and let $G$ be a commutative and quasiprojective $S$-group scheme with unit section $\varepsilon: S \rightarrow G$. Then $R_{S^{\prime} / S}\left(G_{S^{\prime}}\right)$ is a

\footnotetext{
${ }^{4}$ Note that, if $S$ is the spectrum of a field, then every $S$-group scheme of finite type is quasi-projective over $S$ by [7, Proposition A.3.5, p. 486].
} 
commutative and quasi-projective $S$-group scheme with unit section $R_{S^{\prime} / S}\left(\varepsilon_{S^{\prime}}\right)$. See [6, §7.6, Theorem 4, p. 194], [14, II, Corollary 4.5.4] and [7, Proposition A.5.8, p. 513]. Further, by [7, Proposition A.5.2(3), p. 506], the $S$ morphisms $R_{S^{\prime} / S}\left(n_{G_{S^{\prime}}}\right)$ and $n_{R_{S^{\prime} / S}\left(G_{S^{\prime}}\right)}$ can be identified, whence $R_{S^{\prime} / S}\left(G_{S^{\prime}}\right)_{n}=$ $R_{S^{\prime} / S}\left(G_{n, S^{\prime}}\right)$. Now $j_{G, S^{\prime} / S}: G \hookrightarrow R_{S^{\prime} / S}\left(G_{S^{\prime}}\right)$ (2.6) is a closed immersion [7, Proposition A.5.7, p. 510] which induces a closed immersion of commutative and quasi-projective $S$-group schemes $\left(j_{G, S^{\prime} / S}\right)_{n}=j_{G_{n}, S^{\prime} / S}: G_{n} \hookrightarrow R_{S^{\prime} / S}\left(G_{n, S^{\prime}}\right)$. We also note that the composition of $S^{\prime}$-morphisms

$$
G_{S^{\prime}} \stackrel{\left(j_{G, S^{\prime} / S}\right)_{S^{\prime}}}{\longrightarrow} R_{S^{\prime} / S}\left(G_{S^{\prime}}\right)_{S^{\prime}} \stackrel{\theta_{G_{S^{\prime}}, S^{\prime} / S}}{\longrightarrow} G_{S^{\prime}}
$$

equals $n_{G_{S^{\prime}}}=\left(n_{G}\right)_{S^{\prime}}$, where $\theta_{G_{S^{\prime}}, S^{\prime} / S}$ is given by (2.4) (see, e.g., [7, p. 511, lines 23-31]).

Lemma 3.1. If $G$ is a smooth, commutative and quasi-projective S-group scheme with connected fibers, then $R_{S^{\prime} / S}\left(G_{S^{\prime}}\right)$ is (respectively) a smooth, commutative and quasi-projective $S$-group scheme with connected fibers.

Proof. The existence, commutativity and quasi-projectivity of $R_{S^{\prime} / S}\left(G_{S^{\prime}}\right)$ has been noted above. For the smoothness and connectedness of its fibers, see [6, §7.6, Proposition 5, p. 195], [7, Proposition A.5.9, pp. 514] and [9, $\mathrm{VI}_{\mathrm{A}}$, Proposition 2.1.1].

Next let

$$
N_{G, S^{\prime} / S}: R_{S^{\prime} / S}\left(G_{S^{\prime}}\right) \rightarrow G
$$

be the norm morphism defined in [16, XVII, 6.3.13.1 and 6.3.14(a)]. By [16, XVII, Proposition 6.3.17], (3.2) is uniquely determined by the properties of being functorial in $G$, compatible with compositions and arbitrary base changes and by the fact that the composition

$$
n_{G}: G \stackrel{j_{G, S^{\prime} / S}^{\longrightarrow}}{\longrightarrow} R_{S^{\prime} / S}\left(G_{S^{\prime}}\right) \stackrel{N_{G, S^{\prime} S}}{\longrightarrow} G
$$

is the $n$-th power morphism on $G$.

Proposition 3.2. If $G$ is smooth over $S$, then $N_{G, S^{\prime} / S}$ (3.2) is smooth and surjective.

Proof. By [7, Proposition A.5.11(1), p. 516], $\theta_{G_{S^{\prime}}, S^{\prime} / S}: R_{S^{\prime} / S}\left(G_{S^{\prime}}\right)_{S^{\prime}} \rightarrow G_{S^{\prime}}$ (2.4) is a smooth and surjective morphism of $S^{\prime}$-group schemes. Now, if 
we identify $R_{S^{\prime} / S}\left(G_{S^{\prime}}\right)_{S^{\prime}}$ and $R_{S^{\prime \prime} / S^{\prime}}\left(G_{S^{\prime \prime}}\right)$ via [5, (2.40), p. 16], where $S^{\prime \prime}=$ $S^{\prime} \times_{S} S^{\prime}$, then $\left(j_{G, S^{\prime} / S}\right)_{S^{\prime}}: G_{S^{\prime}} \rightarrow R_{S^{\prime} / S}\left(G_{S^{\prime}}\right)_{S^{\prime}}$ is identified with $j_{G_{S^{\prime}}, S^{\prime \prime} / S^{\prime}}$ and $\theta_{G_{S^{\prime}}, S^{\prime} / S}$ is identified with a map $R_{S^{\prime \prime} / S^{\prime}}\left(G_{S^{\prime \prime}}\right) \rightarrow G_{S^{\prime \prime}}$ that has the same properties that characterize $N_{G_{S^{\prime}}, S^{\prime \prime} / S^{\prime}}$. See (3.1) and (3.3). Thus we may identify $\theta_{G_{S^{\prime}}, S^{\prime} / S}$ and $N_{G_{S^{\prime}}, S^{\prime \prime} / S^{\prime}}$, whence $\left(N_{G, S^{\prime} / S}\right)_{S^{\prime}}=N_{G_{S^{\prime}}, S^{\prime \prime} / S^{\prime}}$ is a smooth and surjective morphism of $S^{\prime}$-group schemes. The proposition now follows from [14, $\mathrm{IV}_{4}$, Corollary 17.7.3(ii)] and [15, Proposition 3.6.4, p. 245].

The norm one group scheme associated to $(f, G)$ is the $S$-group scheme

$$
R_{S^{\prime} / S}^{(1)}(G)=\operatorname{Ker}\left[R_{S^{\prime} / S}\left(G_{S^{\prime}} \stackrel{N_{G, S^{\prime} / S}}{\longrightarrow} G\right]\right.
$$

where $N_{G, S^{\prime} / S}$ is the norm morphism (3.2). If $G$ is smooth over $S$, then $R_{S^{\prime} / S}^{(1)}(G)$ is smooth over $S$ by Proposition 3.2. Further, in this case $N_{G, S^{\prime} / S}$ is faithfully flat and the sequence of $S$-group schemes

$$
0 \rightarrow R_{S^{\prime} / S}^{(1)}(G) \rightarrow R_{S^{\prime} / S}\left(G_{S^{\prime}} \stackrel{N_{G, S^{\prime} / S}}{\longrightarrow} G \rightarrow 0\right.
$$

is exact relative to the étale topology on $S$.

Lemma 3.3. If $S^{\prime} \simeq \coprod_{i=1}^{n} S$, then there exist canonical isomorphisms of $S$ group schemes $R_{S^{\prime} / S}(G) \stackrel{\sim}{\rightarrow} G^{n}$ and $R_{S^{\prime} / S}^{(1)}(G) \stackrel{\sim}{\rightarrow} G^{n-1}$.

Proof. The first isomorphism follows from Corollary 2.3 (with $S^{\prime \prime}=S$ there). Under this isomorphism, the norm morphism $N_{G, S^{\prime} / S}: R_{S^{\prime} / S}(G) \rightarrow G$ corresponds to the product morphism $G^{n} \rightarrow G$. See [16, XVII, Proposition 6.3.15(iii)]. The second isomorphism then follows.

Proposition 3.4. Let $S^{\prime} \rightarrow S$ correspond to $B / F$, where $F$ is a field and $B$ is a nonzero, finite and étale $F$-algebra of rank $n \geq 2$. Then $R_{B / F}(G)$ and $R_{B / F}^{(1)}(G)$ are forms of $G^{n}$ and $G^{n-1}$, respectively.

Proof. By [14, $\mathrm{IV}_{4}$, Corollary 17.4.2( $\left.\mathrm{d}^{\prime}\right)$ ], there exists an isomorphism of $F$ algebras $B \simeq \prod_{i=1}^{r} K_{i}$ for some positive integer $r$, where each $K_{i}$ is a finite and separable extension of $F$. Set $f_{i}=\left[K_{i}: F\right]$, so that $n=\operatorname{rank}_{F} B=\sum_{i=1}^{r} f_{i}$. We now choose a separable closure $F^{\mathrm{s}}$ of $F$ containing $K_{i}$ and $F^{\mathrm{s}}$-isomorphisms $K_{i} \otimes_{F} F^{\mathrm{s}} \simeq\left(F^{\mathrm{s}}\right)^{f_{i}}$ for $i=1, \ldots, r$. Then $B \otimes_{F} F^{\mathrm{s}} \simeq \prod_{i=1}^{r}\left(K_{i} \otimes_{F} F^{\mathrm{s}}\right) \simeq$ $\prod_{i=1}^{r}\left(F^{\mathrm{s}}\right)^{f_{i}} \simeq\left(F^{\mathrm{s}}\right)^{n}$, whence $\operatorname{Spec} B \times{ }_{F} \operatorname{Spec} F^{\mathrm{s}} \simeq \coprod_{i=1}^{n} \operatorname{Spec} F^{\mathrm{s}}$. Now Lemma 
3.3 yields isomorphisms of $F^{\mathrm{s}}$-group schemes $R_{B / F}(G) \times{ }_{F} \operatorname{Spec} F^{\mathrm{s}} \simeq G_{F^{\mathrm{s}}}^{n}$ and $R_{B / F}^{(1)}(G) \times_{F} \operatorname{Spec} F^{\mathrm{s}} \simeq G_{F^{\mathrm{s}}}^{n-1}$, as claimed.

Lemma 3.5. Let $S^{\prime} \rightarrow S$ correspond to $B / F$, where $F$ is a field and $B$ is a finite F-algebra. Let $0 \rightarrow G^{\prime} \rightarrow G \rightarrow G^{\prime \prime} \rightarrow 0$ be an exact sequence of smooth, commutative and quasi-projective F-group schemes. Then the given sequence induces exact sequences of smooth, commutative and quasi-projective F-group schemes

$$
0 \rightarrow R_{B / F}\left(G_{B}^{\prime}\right) \rightarrow R_{B / F}\left(G_{B}\right) \rightarrow R_{B / F}\left(G_{B}^{\prime \prime}\right) \rightarrow 0
$$

and

$$
0 \rightarrow R_{B / F}^{(1)}\left(G^{\prime}\right) \rightarrow R_{B / F}^{(1)}(G) \rightarrow R_{B / F}^{(1)}\left(G^{\prime \prime}\right) \rightarrow 0 .
$$

Proof. The first sequence is exact by [7, Proposition A.5.4(3), p. 508]. The second sequence follows by applying the snake lemma to the following exact and commutative diagram in $(\operatorname{Spec} F) \tilde{f l}$, whose vertical arrows are surjections by Proposition 3.2.

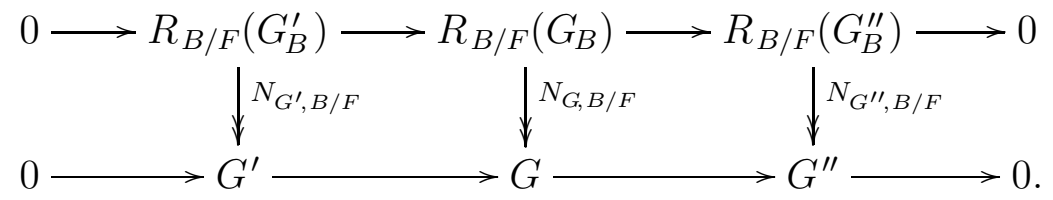

Proposition 3.6. Let $S^{\prime} \rightarrow S$ correspond to $B / F$, where $F$ is a field and $B$ is a nonzero, finite and étale $F$-algebra. If $G$ is a torus (respectively, abelian variety, semiabelian variety) over $F$, then $R_{B / F}(G)$ and $R_{B / F}^{(1)}(G)$ are tori (respectively, abelian varieties, semiabelian varieties) over $F$.

Proof. If $G$ is a torus (respectively, abelian variety) over $F$, then so also are $R_{B / F}(G)$ and $R_{B / F}^{(1)}(G)$ by Proposition (3.4). Now, if $G$ is a semiabelian variety over $F$ given as an extension $1 \rightarrow T \rightarrow G \rightarrow A \rightarrow 1$, where $T$ (respectively, $A$ ) is a torus (respectively, abelian variety) over $F$, then Lemma 3.5 shows that $R_{B / F}(G)$ and $R_{B / F}^{(1)}(G)$ are semiabelian varieties over $F$ given as extensions

$$
0 \rightarrow R_{B / F}(T) \rightarrow R_{B / F}(G) \rightarrow R_{B / F}(A) \rightarrow 0
$$

and

$$
0 \rightarrow R_{B / F}^{(1)}(T) \rightarrow R_{B / F}^{(1)}(G) \rightarrow R_{B / F}^{(1)}(A) \rightarrow 0
$$


Lemma 3.7. Let $S^{\prime} \rightarrow S$ correspond to $K / F$, where $F$ is a field and $K$ is a finite and purely inseparable extension of $F$. If $G$ is a smooth, commutative and quasi-projective F-group scheme, then $R_{K / F}^{(1)}(G)$ is a smooth, connected and unipotent F-group scheme.

Proof. The smoothness of $R_{K / F}^{(1)}(G)$ has already been noted. By the proof of Proposition [3.2, the maps $\left(N_{G, K / F}\right)_{K}$ and $\theta_{G_{K}, K / F}$ can be identified. Now, by [7, Proposition A.5.11(2), p. 517], $\operatorname{Ker} \theta_{G_{K}, K / F}$ is connected and unipotent. Thus $R_{K / F}^{(1)}(G)_{K}$ is connected and unipotent over $K$, whence $R_{K / F}^{(1)}(G)$ is connected and unipotent over $F$.

Lemma 3.8. Let $S^{\prime} \rightarrow S$ correspond to a finite field extension $K / F$ and let $G$ be a smooth, commutative, connected and quasi-projective $F$-group scheme. Then $R_{K / F}^{(1)}(G)$ is smooth and connected.

Proof. Let $L$ denote the separable closure of $F$ in $K$. By Proposition 3.2 , $N_{G_{L}, K / L}: R_{K / L}\left(G_{K}\right) \rightarrow G_{L}$ is a smooth surjection of quasi-projective $L$-group schemes. Thus, by [7, Corollary A.5.4(3), p. 507] and the transitivity of Weil restrictions [5, (2.41), p. 16], there exists a canonical exact sequence of smooth and quasi-projective $F$-group schemes

$$
0 \rightarrow R_{L / F}\left(R_{K / L}^{(1)}\left(G_{L}\right)\right) \rightarrow R_{K / F}\left(G_{K}\right) \rightarrow R_{L / F}\left(G_{L}\right) \rightarrow 0
$$

where the third map above can be identified with $R_{L / F}\left(N_{G_{L}, K / L}\right)$. We now apply Lemma 2.1 (in the abelian category $(\operatorname{Spec} F) \tilde{\text { fl }}$ ) to the pair of $F$-morphisms

$$
R_{K / F}\left(G_{K}\right) \rightarrow R_{L / F}\left(G_{L}\right) \rightarrow G
$$

whose composition is $N_{G, L / F} \circ R_{L / F}\left(N_{G_{L}, K / L}\right)=N_{G, K / F}$. Since the kernel of the first map in (3.5) is $R_{L / F}\left(R_{K / L}^{(1)}\left(G_{L}\right)\right)$ by the exactness of (3.4), we obtain an exact sequence of smooth and quasi-projective $F$-group schemes

$$
0 \rightarrow R_{L / F}\left(R_{K / L}^{(1)}\left(G_{L}\right)\right) \rightarrow R_{K / F}^{(1)}(G) \rightarrow R_{L / F}^{(1)}(G) \rightarrow 0 .
$$

By Lemma 3.7, $R_{K / L}^{(1)}\left(G_{L}\right)$ is smooth, connected and unipotent over $L$, whence $R_{L / F}\left(R_{K / L}^{(1)}\left(G_{L}\right)\right)$ is smooth, connected and unipotent over $F$ by [7, Proposition A.5.9, p. 514] and [22, Proposition A.3.7, p. 84]. On the other hand, $R_{L / F}^{(1)}(G)$ 
is connected since it is a form of $G^{[L: F]-1}$ by Proposition 3.4. The lemma now follows from the exactness of (3.6) and [5, Lemma 2.55].

Proposition 3.9. Let $S^{\prime} \rightarrow S$ correspond to $B / F$, where $F$ is a field and $B$ is a local and finite $F$-algebra. Let $G$ be a smooth, commutative, connected and quasi-projective F-group scheme. Then $R_{B / F}^{(1)}(G)$ is smooth and connected.

Proof. Let $K$ be the residue field of $B$. As in the proof of Lemma 3.8, there exists a canonical exact sequence of smooth and quasi-projective $F$-group schemes

$$
0 \rightarrow R_{K / F}\left(R_{B / K}^{(1)}\left(G_{K}\right)\right) \rightarrow R_{B / F}^{(1)}(G) \rightarrow R_{K / F}^{(1)}(G) \rightarrow 0,
$$

where the right-hand group above is connected by Lemma 3.8 . Now, by 5 , Proposition 20.2], $R_{B / K}^{(1)}\left(G_{K}\right)$ is smooth, connected and unipotent over $K$. The rest of the proof is similar to the last part of the proof of Lemma 3.8 .

Proposition 3.10. Let $S^{\prime} \rightarrow S$ be a finite and faithfully flat morphism of locally noetherian schemes and let $G$ be a smooth, commutative and quasiprojective $S$-group scheme with connected fibers. Then $R_{S^{\prime} / S}(G)$ and $R_{S^{\prime} / S}^{(1)}(G)$ are smooth and commutative with connected fibers.

Proof. By Lemma 3.1, we need only consider $R_{S^{\prime} / S}^{(1)}(G)$. Let $s$ be a point of $S$ with residue field $k(s)$ and write $S^{\prime} \times_{S} \operatorname{Spec} k(s)=\operatorname{Spec} B(s)$. By [2, Exercise 3 , p. 92 and Theorem 8.7, p. 90], there exists an isomorphism of $k(s)$-algebras $B(s) \simeq \prod_{i=1}^{m} B(s)_{i}$, where each $B(s)_{i}$ is a local and finite $k(s)$-algebra. Thus, by Proposition 2.2 , there exist isomorphisms of $k(s)$-group schemes

$$
R_{S^{\prime} / S}^{(1)}(G)_{s} \simeq R_{B(s) / k(s)}^{(1)}\left(G_{s}\right) \simeq \prod_{i=1}^{m} R_{B(s)_{i} / k(s)}^{(1)}\left(G_{s}\right) .
$$

The proposition is now immediate from Proposition 3.9 and $\left[9, \mathrm{VI}_{\mathrm{A}}\right.$, Proposition 2.1.1].

Definition 3.11. The pair $(f, G)$ is called admissible if

(i) $f: S^{\prime} \rightarrow S$ is a finite and faithfully flat morphism of locally noetherian schemes of constant rank $n \geq 2$,

(ii) $G$ is a smooth, commutative and quasi-projective $S$-group scheme with connected fibers, and

(iii) for every point $s \in S$ such that $\operatorname{char} k(s)$ divides $n$, 
(iii.1) $G_{k(s)}$ is a semiabelian $k(s)$-variety, and

(iii.2) $f_{s}: S^{\prime} \times{ }_{S} \operatorname{Spec} k(s) \rightarrow \operatorname{Spec} k(s)$ is étale.

Proposition 3.12. Assume that $(f, G)=\left(S^{\prime} / S, G\right)$ is admissible (see Definition 3.11) and let $n \geq 2$ be the rank of $f$. If $H=G, R_{S^{\prime} / S}\left(G_{S^{\prime}}\right)$ or $R_{S^{\prime} / S}^{(1)}(G)$, then $n: H \rightarrow H$ is faithfully flat and locally of finite presentation.

Proof. It was shown in [12, Proposition 3.3] that conditions (ii) (minus the quasi-projectivity hypothesis) and (iii.1) of Definition 3.11 imply the proposition when $H=G$. Thus, to establish the proposition when $H=R_{S^{\prime} / S}\left(G_{S^{\prime}}\right)$ or $H=R_{S^{\prime} / S}^{(1)}(G)$, it suffices to check that conditions (ii) (minus the quasiprojectivity hypothesis) and (iii.1) of Definition 3.11 hold true when $G$ is replaced with $R_{S^{\prime} / S}\left(G_{S^{\prime}}\right)$ or $R_{S^{\prime} / S}^{(1)}(G)$. For condition (ii) (minus the quasiprojectivity hypothesis), see Proposition 3.10. Now let $s \in S$ be such that char $k(s)$ divides $n$. Then $R_{S^{\prime} / S}(G)_{s} \simeq R_{B(s) / k(s)}\left(G_{s}\right)$ and similarly for $R_{S^{\prime} / S}^{(1)}(G)$, where $B(s)$ is a finite and étale $k(s)$-algebra by Definition 3.11(iii.2). Since $G_{s}$ is a semiabelian $k(s)$-variety by Definition [3.11(iii.1), $R_{B(s) / k(s)}\left(G_{s}\right)$ and $R_{B(s) / k(s)}^{(1)}\left(G_{s}\right)$ are semiabelian $k(s)$-varieties by Proposition 3.6, i.e., $R_{S^{\prime} / S}\left(G_{S^{\prime}}\right)$ and $R_{S^{\prime} / S}^{(1)}(G)$ satisfy Definition $3.11($ iii.1). The proof is now complete.

\section{Proof of the MAIN THEOREM}

Let $f: S^{\prime} \rightarrow S$ be a finite and faithfully flat morphism of locally noetherian schemes of constant rank $n \geq 2$ and let $G$ be a commutative and quasiprojective $S$-group scheme.

The Cartan-Leray spectral sequence associated to $(f, G)$ relative to the $\tau$ topology

$$
H^{r}\left(S_{\tau}, R^{s} f_{*}\left(G_{S^{\prime}}\right)\right) \Longrightarrow H^{r+s}\left(S_{\tau}^{\prime}, G\right)
$$

induces edge morphisms $e_{\tau}^{(r)}: H^{r}\left(S_{\tau}, R_{S^{\prime} / S}\left(G_{S^{\prime}}\right)\right) \rightarrow H^{r}\left(S_{\tau}^{\prime}, G\right)$ for every $r \geq 0$ [26, Proposition 2.3.1, p. 14]. The map $e_{\tau}^{(0)}$ is the isomorphism (2.5) for $X^{\prime}=$ $G_{S^{\prime}}$. Further, by [26, Theorem 6.4.2(ii), p. 128], the maps $e_{\text {ét }}^{(r)}$ are isomorphisms for every $r \geq 0$. Note, however, that the maps $e_{\mathrm{fl}}^{(r)}$ are not isomorphisms in general [9, XXIV, Remarks 8.5].

Now consider

$$
j_{G, \tau}^{(r)}=H^{r}\left(S_{\tau}, j_{G, S^{\prime} / S}\right): H^{r}\left(S_{\tau}, G\right) \rightarrow H^{r}\left(S_{\tau}, R_{S^{\prime} / S}\left(G_{S^{\prime}}\right)\right),
$$


where $j_{G, S^{\prime} / S}$ is the map (2.6) associated to $X=G$. The composition

$$
H^{r}\left(S_{\text {ét }}, G\right) \stackrel{j_{G, \text { ét }}^{(r)}}{\longrightarrow} H^{r}\left(S_{\text {ét }}, R_{S^{\prime} / S}\left(G_{S^{\prime}}\right)\right) \stackrel{e_{\text {et }}^{(r)}}{\sim} H^{r}\left(S_{\text {ét }}^{\prime}, G\right)
$$

is the natural $r$-th restriction map in étale cohomology

$$
\operatorname{Res}_{G}^{(r)}: H^{r}\left(S_{\text {ét }}, G\right) \rightarrow H^{r}\left(S_{\text {ét }}^{\prime}, G\right) .
$$

We now consider

$$
N_{G, \tau}^{(r)}=H^{r}\left(S_{\tau}, N_{G, S^{\prime} / S}\right): H^{r}\left(S_{\tau}, R_{S^{\prime} / S}\left(G_{S^{\prime}}\right)\right) \rightarrow H^{r}\left(S_{\tau}, G\right),
$$

where $N_{G, S^{\prime} / S}$ is the norm morphism (3.2). By [16, XVII, Example 6.3.18], $N_{G, \tau}^{(0)}$ can be identified with a map

$$
N_{S^{\prime} / S}: G\left(S^{\prime}\right) \rightarrow G(S)
$$

that agrees with the usual norm map if $G=\mathbb{G}_{m, S}$. The composition

$$
\operatorname{Cores}_{G}^{(r)}: H^{r}\left(S_{\text {ét }}^{\prime}, G\right) \stackrel{\left(e_{\text {êt }}^{(r)}\right)^{-1}}{\sim} H^{r}\left(S_{\text {ét }}, R_{S^{\prime} / S}\left(G_{S^{\prime}}\right)\right) \stackrel{N_{G, \text { ét }}^{(r)}}{\longrightarrow} H^{r}\left(S_{\text {ét }}, G\right)
$$

is the $r$-th corestriction map in étale cohomology. For $r=0$ the maps (4.2) and (4.3) will be identified, i.e.,

$$
\operatorname{Cores}_{G}^{(0)}=N_{S^{\prime} / S}
$$

Further, the maps $\operatorname{Cores}_{\mathbb{G}_{m, S}}^{(i)}$ for $i=1$ and 2 will both be denoted by Cores $_{S^{\prime} / S}$ since no ambiguity will result from this choice of notation. If $n \geq 1$ is an integer, we will write $\operatorname{Res}_{G, n}^{(r)}$ and $\operatorname{Cores}_{G, n}^{(r)}$ for $\left(\operatorname{Res}_{G}^{(r)}\right)_{n}$ and $\left(\operatorname{Cores}_{G}^{(r)}\right)_{n}$, respectively. The following equalities hold for all $r \geq 0$ and all $n \geq 1$ :

$$
\begin{aligned}
& \operatorname{Ker} j_{G, \text { ét }}^{(r)}=\operatorname{Ker} \operatorname{Res}_{G}^{(r)} \\
& \operatorname{Ker}\left(j_{G \text {,ét }}^{(r)} / n\right)=\operatorname{Ker}\left(\operatorname{Res}_{G}^{(r)} / n\right) \\
& \operatorname{Coker}\left(\left(N_{G \text {,ét }}^{(r)}\right)_{n}\right)=\text { Coker Cores }_{G, n}^{(r)} \\
& \operatorname{Coker}\left(\left(j_{G, \text { ét }}^{(r)}\right)_{n}\right)=\operatorname{Coker} \operatorname{Res}_{G, n}^{(r)} \\
& \operatorname{Ker}\left(N_{G, \text { ét }}^{(r)}\right)_{n}=\operatorname{Ker} \operatorname{Cores}_{G, n}^{(r)} .
\end{aligned}
$$

Next, by (3.3), the composition

$$
H^{r}\left(S_{\tau}, G\right) \stackrel{j_{G, \tau}^{(r)}}{\longrightarrow} H^{r}\left(S_{\tau}, R_{S^{\prime} / S}\left(G_{S^{\prime}}\right)\right) \stackrel{N_{G, \tau}^{(r)}}{\longrightarrow} H^{r}\left(S_{\tau}, G\right)
$$


is the multiplication by $n$ map on $H^{r}\left(S_{\tau}, G\right)$. Thus $\operatorname{Ker} j_{G, \tau}^{(r)}$ and Coker $N_{G, \tau}^{(r)}$ are $n$-torsion abelian groups and (2.1) yields

$$
\operatorname{Ker}\left(j_{G, \tau}^{(r)}\right)_{n}=\operatorname{Ker} j_{G, \tau}^{(r)}
$$

and

$$
\operatorname{Coker}\left(N_{G, \tau}^{(r)} / n\right)=\operatorname{Coker} N_{G, \tau}^{(r)} .
$$

Now observe that (4.10) induces three complexes (in degrees 0,1 and 2) of $n$-torsion abelian groups, namely

$$
C_{/ n}^{\bullet}(r, G)=\left(H^{r}\left(S_{\text {ét }}, G\right) / n \stackrel{j_{G, \text { ét }}^{(r)} / n}{\longrightarrow} H^{r}\left(S_{\text {ét }}, R_{S^{\prime} / S}\left(G_{S^{\prime}}\right)\right) / n \stackrel{N_{G, \text { ét }}^{(r)} / n}{\longrightarrow} H^{r}\left(S_{\text {ét }}, G\right) / n\right),
$$

$$
C^{\bullet}\left(r, G_{n}\right)=\left(H^{r}\left(S_{\mathrm{fl}}, G_{n}\right) \stackrel{j_{G_{n}, \mathrm{fl}}^{(r)}}{\longrightarrow} H^{r}\left(S_{\mathrm{fl}}, R_{S^{\prime} / S}\left(G_{n, S^{\prime}}\right)\right) \stackrel{N_{G_{n}, \mathrm{fl}}^{(r)}}{\longrightarrow} H^{r}\left(S_{\mathrm{fl}}, G_{n}\right)\right),
$$

and

$$
C_{n}^{\bullet}(r, G)=\left(H^{r}\left(S_{\text {ét }}, G\right)_{n} \stackrel{\left(j_{G, \text { ét }}^{(r)}\right)_{n}}{\longrightarrow} H^{r}\left(S_{\text {ét }}, R_{S^{\prime} / S}\left(G_{S^{\prime}}\right)\right)_{n} \stackrel{\left(N_{G, \text { ét }}^{(r)}\right)_{n}}{\longrightarrow} H^{r}\left(S_{\text {ét }}, G\right)_{n}\right) .
$$

Using (4.5)-(4.7) and (4.11)-(4.12), we have

$$
\begin{aligned}
& H^{0}\left(C_{/ n}^{\bullet}(r, G)\right)=\operatorname{Ker}\left(\operatorname{Res}_{G}^{(r)} / n\right) \\
& H^{0}\left(C^{\bullet}\left(r, G_{n}\right)\right)=\operatorname{Ker} j_{G_{n}, \mathrm{fl}}^{(r)} \\
& H^{0}\left(C_{n}^{\bullet}(r, G)\right)=\operatorname{Ker} \operatorname{Res}_{G}^{(r)} \\
& H^{2}\left(C_{/ n}^{\bullet}(r, G)\right)={\text { Coker } \operatorname{Cores}_{G}^{(r)}}^{(r)} \\
& H^{2}\left(C^{\bullet}\left(r, G_{n}\right)\right)=\operatorname{Coker} N_{G_{n}, \mathrm{fl}}^{(r)} \\
& H^{2}\left(C_{n}^{\bullet}(r, G)\right)=\operatorname{Coker}_{\operatorname{Cores}_{G, n}^{(r)}} .
\end{aligned}
$$

We now define

$$
\Psi_{N}(n, G)=\left\{x \in G\left(S^{\prime}\right): N_{S^{\prime} / S}(x) \in G(S)^{n}\right\}
$$


where $N_{S^{\prime} / S}$ is the map (4.2). Then $\Psi_{N}(n, G)$ is a subgroup of $G\left(S^{\prime}\right)$ which contains $G(S) G\left(S^{\prime}\right)^{n}$ and we have

$$
H^{1}\left(C_{/ n}^{\bullet}(0, G)\right)=\frac{\Psi_{N}(n, G)}{G(S) G\left(S^{\prime}\right)^{n}} .
$$

We now assume that $(f, G)$ is an admissible pair (see Definition 3.11).

Since $(f, G)$ is admissible, Proposition 3.12 yields an exact and commutative diagram in $S_{\mathrm{fl}}^{\sim}$

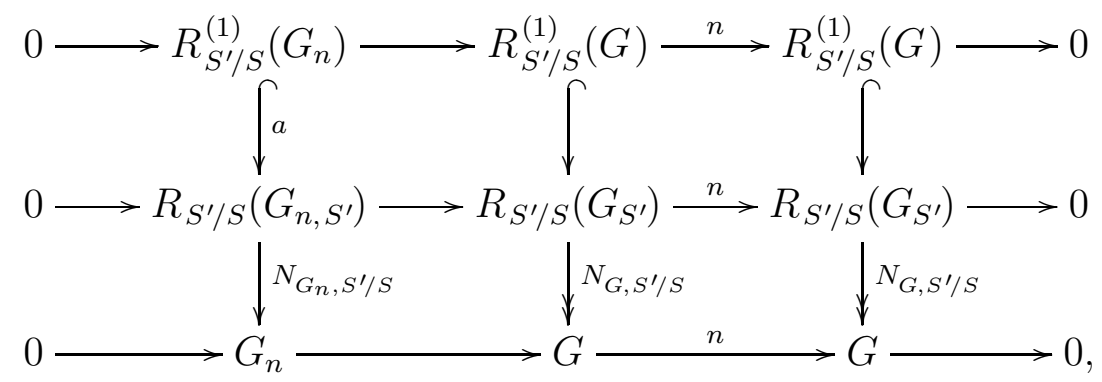

where $a$ is the inclusion morphism. A diagram chase (or an application of the snake lemma to the bottom half of the above diagram) shows that $N_{G_{n}, S^{\prime} / S}$ is surjective, whence

$$
0 \rightarrow R_{S^{\prime} / S}^{(1)}\left(G_{n}\right) \stackrel{a}{\longrightarrow} R_{S^{\prime} / S}\left(G_{n, S^{\prime}}\right) \stackrel{N_{G_{n}, S^{\prime} / S}}{\longrightarrow} G_{n} \rightarrow 0
$$

is an exact sequence in $S_{\mathrm{fl}}^{\sim}$. On the other hand, by (3.3) applied to $G_{n}$,

$$
G_{n} \stackrel{j_{G_{n}, S^{\prime} / S}}{\longrightarrow} R_{S^{\prime} / S}\left(G_{n, S^{\prime}}\right) \stackrel{N_{G_{n}, S^{\prime} / S}}{\longrightarrow} G_{n}
$$

is a complex of commutative and quasi-projective $S$-group schemes. Thus there exists a closed immersion of commutative $S$-group schemes

$$
b: G_{n} \rightarrow R_{S^{\prime} / S}^{(1)}\left(G_{n}\right) .
$$

We will write

$$
G(n)=R_{S^{\prime} / S}^{(1)}\left(G_{n}\right) / G_{n}
$$

for the cokernel of $b$ in $S_{\mathrm{fl}}^{\sim}$. Note that $G(n)$ is represented by an $S$-group scheme if $G_{n}$ is flat over $S$ and $\operatorname{dim} S \leq 1$ [1, Theorem 4.C, p. 53]. Now, by 
definition of $G(n)$, there exists a canonical exact sequence in $S_{\mathrm{fl}}$

$$
0 \rightarrow G_{n} \stackrel{b}{\longrightarrow} R_{S^{\prime} / S}^{(1)}\left(G_{n}\right) \stackrel{q}{\longrightarrow} G(n) \rightarrow 0,
$$

where $q$ is the projection and $b$ is the inclusion map. Note that $a \circ b=j_{G_{n}, S^{\prime} / S}$. Now, for every $r \geq 0$, consider

$$
\begin{aligned}
a^{(r)} & =H^{r}\left(S_{\mathrm{fl}}, a\right): H^{r}\left(S_{\mathrm{fl}}, R_{S^{\prime} / S}^{(1)}\left(G_{n}\right)\right) \rightarrow H^{r}\left(S_{\mathrm{fl}}, R_{S^{\prime} / S}\left(G_{n, S^{\prime}}\right)\right) \\
b^{(r)} & =H^{r}\left(S_{\mathrm{f}}, b\right): H^{r}\left(S_{\mathrm{fl}}, G_{n}\right) \rightarrow H^{r}\left(S_{\mathrm{fl}}, R_{S^{\prime} / S}^{(1)}\left(G_{n}\right)\right) \\
q^{(r)} & =H^{r}\left(S_{\mathrm{f}}, q\right): H^{r}\left(S_{\mathrm{fl}}, R_{S^{\prime} / S}^{(1)}\left(G_{n}\right)\right) \rightarrow H^{r}\left(S_{\mathrm{fl}}, G(n)\right) .
\end{aligned}
$$

Then the sequences (4.25) and (4.28) induce exact sequences of abelian groups (4.29)

$$
\cdots \rightarrow H^{r-1}\left(S_{\mathrm{fl}}, R_{S^{\prime} / S}\left(G_{n, S^{\prime}}\right)\right) \stackrel{N_{G_{n}, \mathrm{fl}}^{(r-1)}}{\longrightarrow} H^{r-1}\left(S_{\mathrm{fl}}, G_{n}\right) \stackrel{\delta^{(r-1)}}{\longrightarrow} H^{r}\left(S_{\mathrm{fl}}, R_{S^{\prime} / S}^{(1)}\left(G_{n}\right)\right) \stackrel{a^{(r)}}{\longrightarrow} \ldots
$$

and

$$
\begin{aligned}
\cdots \rightarrow H^{r}\left(S_{\mathrm{fl}}, G_{n}\right) \stackrel{b^{(r)}}{\longrightarrow} H^{r}\left(S_{\mathrm{fl}}, R_{S^{\prime} / S}^{(1)}\left(G_{n}\right)\right) & \stackrel{q^{(r)}}{\longrightarrow} H^{r}\left(S_{\mathrm{fl}}, G(n)\right) \\
& \stackrel{\partial^{(r)}}{\longrightarrow} H^{r+1}\left(S_{\mathrm{fl}}, G_{n}\right) \rightarrow \ldots,
\end{aligned}
$$

where the maps $\delta^{(r)}$ and $\partial^{(r)}$ are connecting morphisms induced by (4.25) and (4.28), respectively. Note that, since $a^{(r)} \circ b^{(r)}=j_{G_{n}, \mathrm{fl}}^{(r)}$ and $\operatorname{Ker} q^{(r)}=\operatorname{Im} b^{(r)}$, we have

$$
a^{(r)}\left(\operatorname{Ker} q^{(r)}\right)=\operatorname{Im} j_{G_{n}, \mathrm{fl}}^{(r)}
$$

Now, for every $r \geq 1$, let

$$
\gamma^{(r)}: H^{r-1}\left(S_{\mathrm{fl}}, G_{n}\right) \rightarrow H^{r}\left(S_{\mathrm{f}}, G(n)\right)
$$

be the composition

$$
H^{r-1}\left(S_{\mathrm{fl}}, G_{n}\right) \stackrel{\delta^{(r-1)}}{\longrightarrow} H^{r}\left(S_{\mathrm{fl}}, R_{S^{\prime} / S}^{(1)}\left(G_{n}\right)\right) \stackrel{q^{(r)}}{\longrightarrow} H^{r}\left(S_{\mathrm{fl}}, G(n)\right) .
$$

By the exactness of (4.29) and (4.30), there exists a canonical isomorphism of abelian groups

$$
\operatorname{Ker} a^{(r)} / \operatorname{Ker} a^{(r)} \cap \operatorname{Ker} q^{(r)} \stackrel{\sim}{\rightarrow} q^{(r)}\left(\operatorname{Ker} a^{(r)}\right)=q^{(r)}\left(\operatorname{Im} \delta^{(r)}\right)=\operatorname{Im} \gamma^{(r)}
$$


Next consider the complex

$$
\Gamma_{n}^{\bullet}(r, G)=\left(H^{r-1}\left(S_{\mathrm{fl}}, G_{n}\right) \stackrel{\gamma^{(r)}}{\longrightarrow} H^{r}\left(S_{\mathrm{fl}}, G(n)\right) \stackrel{\partial^{(r)}}{\longrightarrow} H^{r+1}\left(S_{\mathrm{fl}}, G_{n}\right)\right) .
$$

Lemma 4.1. For every $r \geq 1$, there exists a canonical isomorphism of $n$ torsion abelian groups

$$
H^{1}\left(C^{\bullet}\left(r, G_{n}\right)\right) \simeq H^{1}\left(\Gamma_{n}^{\bullet}(r, G)\right),
$$

where the complexes $C^{\bullet}\left(r, G_{n}\right)$ and $\Gamma_{n}^{\bullet}(r, G)$ are given by (4.14) and (4.34), respectively.

Proof. By the exactness of (4.30) and the identity (4.31), the following diagram is exact and commutative

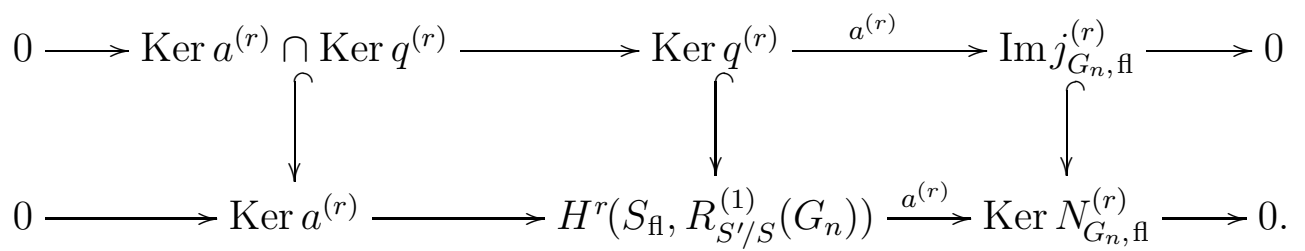

The above diagram induces the top row of the following commutative diagram of $n$-torsion abelian groups with exact rows

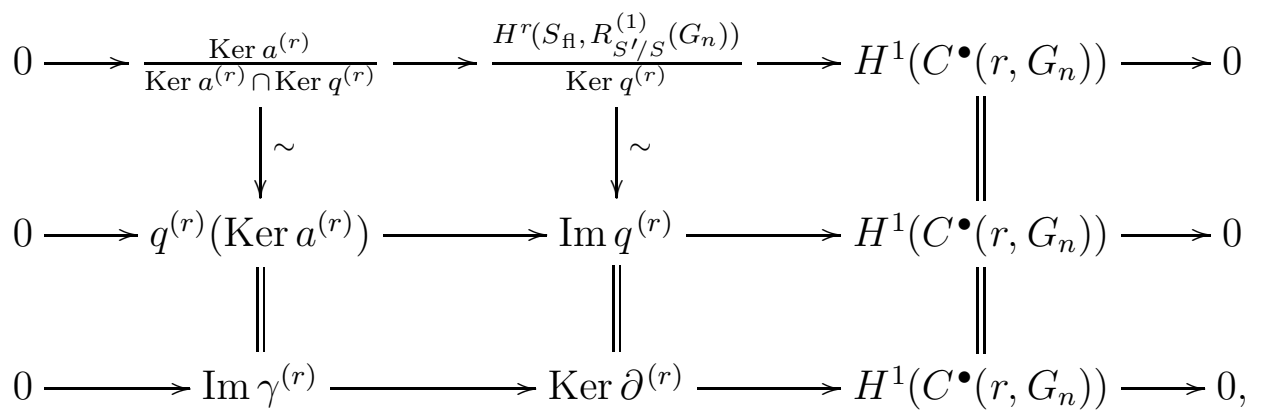

where the left-hand vertical composition is the isomorphism (4.33) and the top middle vertical isomorphism comes from (4.30). Thus we obtain a canonical isomorphism Ker $\partial^{(r)} / \operatorname{Im} \gamma^{(r)}=H^{1}\left(\Gamma_{n}^{\bullet}(r, G)\right) \stackrel{\sim}{\rightarrow} H^{1}\left(C^{\bullet}\left(r, G_{n}\right)\right)$, as claimed.

Next, the middle and bottom rows of diagram (4.24) induce short exact sequences of abelian groups for every integer $r \geq 1$

$$
H^{r-1}\left(S_{\text {ét }}, R_{S^{\prime} / S}\left(G_{S^{\prime}}\right)\right) / n \hookrightarrow H^{r}\left(S_{\mathrm{fl}}, R_{S^{\prime} / S}\left(G_{n, S^{\prime}}\right)\right) \rightarrow H^{r}\left(S_{\text {ét }}, R_{S^{\prime} / S}\left(G_{S^{\prime}}\right)\right)_{n}
$$


and

$$
0 \rightarrow H^{r-1}\left(S_{\text {ét }}, G\right) / n \rightarrow H^{r}\left(S_{\text {ff }}, G_{n}\right) \rightarrow H^{r}\left(S_{\text {ét }}, G\right)_{n} \rightarrow 0 .
$$

The preceding sequences fit into the following exact and commutative diagram of abelian groups

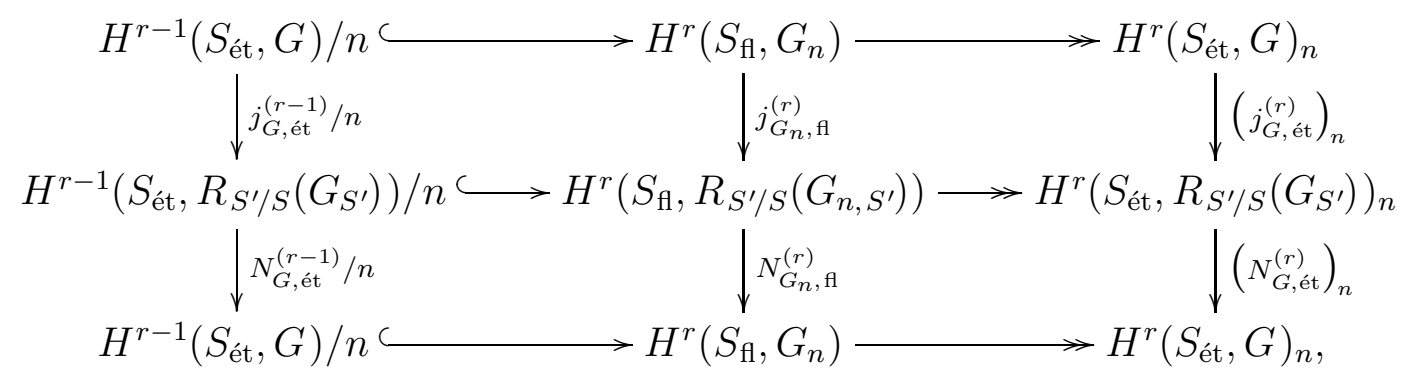

i.e., there exists a canonical exact sequence of complexes of abelian groups

$$
0 \rightarrow C_{/ n}^{\bullet}(r-1, G) \rightarrow C^{\bullet}\left(r, G_{n}\right) \rightarrow C_{n}^{\bullet}(r, G) \rightarrow 0,
$$

where the left-hand, middle and right-hand complexes are given by (4.13), (4.14) and (4.15), respectively. We can now state the main theorem of the paper.

Theorem 4.2. Assume that the pair $(f, G)$ is admissible (see Definition 3.11) and let $n \geq 2$ be the rank of $f$. For every integer $r \geq 1$, there exists a canonical exact sequence of $n$-torsion abelian groups

$$
\begin{aligned}
0 & \rightarrow \operatorname{Ker}\left(\operatorname{Res}_{G}^{(r-1)} / n\right) \rightarrow \operatorname{Ker} j_{G_{n}, \mathrm{fl}}^{(r)} \rightarrow \operatorname{Ker}_{\operatorname{Res}_{G}^{(r)}}^{(r)} \\
& \rightarrow H^{1}\left(C_{/ n}^{\bullet}(r-1, G)\right) \rightarrow H^{1}\left(\Gamma_{n}^{\bullet}(r, G)\right) \rightarrow H^{1}\left(C_{n}^{\bullet}(r, G)\right) \\
& \rightarrow \operatorname{Coker} \operatorname{Cores}_{G}^{(r-1)} \rightarrow \operatorname{Coker} N_{G_{n}, \mathrm{fl}}^{(r)} \rightarrow \operatorname{Coker} \operatorname{Cores}_{G, n}^{(r)} \rightarrow 0,
\end{aligned}
$$

where the complexes $C_{/ n}^{\bullet}(r-1, G), \Gamma_{n}^{\bullet}(r, G)$ and $C_{n}^{\bullet}(r, G)$ are given by (4.13), (4.34) and (4.15), respectively.

Proof. The sequence of the theorem is derived from the cohomology sequence induced by (4.35) using the identities (4.16)-(4.21) and the isomorphism of Lemma 4.1. 
Corollary 4.3. Under the hypotheses of the theorem, there exists a canonical exact sequence of $n$-torsion abelian groups

$$
\begin{aligned}
0 & \rightarrow G(S) \cap G\left(S^{\prime}\right)^{n} / G(S)^{n} \rightarrow \check{H}^{1}\left(S^{\prime} / S, G_{n}\right) \rightarrow \operatorname{Ker}_{\operatorname{Res}_{G}^{(1)}} \\
& \rightarrow \Psi_{N}(n, G) / G(S) G\left(S^{\prime}\right)^{n} \rightarrow H^{1}\left(\Gamma_{n}^{\bullet}(1, G)\right) \rightarrow H^{1}\left(C_{n}^{\bullet}(1, G)\right) \\
& \rightarrow G(S) / N_{S^{\prime} / S}\left(G\left(S^{\prime}\right)\right) \rightarrow \text { Coker } N_{G_{n}, \mathrm{fl}}^{(1)} \rightarrow \text { Coker } \operatorname{Cores}_{G, n}^{(1)} \rightarrow 0,
\end{aligned}
$$

where $\check{H}^{1}\left(S^{\prime} / S, G_{n}\right)$ is the first $\check{C}$ ech cohomology group of $G_{n}$ relative to the fppf covering $S^{\prime} / S$ and $\Psi_{N}(n, G)=\left\{x \in G\left(S^{\prime}\right): N_{S^{\prime} / S}(x) \in G(S)^{n}\right\}$.

Proof. Set $r=1$ in the theorem and use the identities (4.4), (4.22) and (4.23) together with the canonical isomorphism $\operatorname{Ker} j_{G_{n}, \mathrm{fl}}^{(r)} \simeq \breve{H}^{1}\left(S^{\prime} / S, G_{n}\right)$ of [12, Proposition 2.1].

\section{Quadratic Galois Coverings}

Let $(f, G)$ be an admissible pair such that $f$ is étale and let $\Delta$ be a finite group of order $n \geq 2$ which acts on $S^{\prime} / S$ from the right. Then $f$ is called a Galois covering with Galois group $\Delta$ if the canonical map

$$
\coprod_{\delta \in \Delta} S^{\prime} \rightarrow S^{\prime} \times_{S} S^{\prime},(x, \delta) \mapsto(x, x \delta),
$$

is an isomorphism of $S$-schemes. See [13, V, Proposition 2.6 and Definition 2.8]. In this case $n=\operatorname{rank} f$ and there exists a canonical isomorphism of abelian groups

$$
\check{H}^{1}\left(S^{\prime} / S, G_{n}\right) \simeq H^{1}\left(\Delta, G\left(S^{\prime}\right)_{n}\right),
$$

where $G\left(S^{\prime}\right)_{n}$ is a left $\Delta$-module via the given right action of $\Delta$ on $S^{\prime} / S$. See [20, III, Example 2.6, p. 99].

By (5.1) and Corollary 2.3 (with $S^{\prime \prime}=S^{\prime}$ there), there exists a canonical isomorphism of $S^{\prime}$-schemes

$$
R_{S^{\prime} / S}^{(1)}\left(G_{n}\right)_{S^{\prime}} \stackrel{\sim}{\rightarrow} G_{n, S^{\prime}}^{n-1}
$$

(see the proof of Lemma 3.3). Under the preceding isomorphism, the closed immersion $b_{S^{\prime}}: G_{n, S^{\prime}} \hookrightarrow R_{S^{\prime} / S}^{(1)}\left(G_{n}\right)_{S^{\prime}}$ induced by (4.26) corresponds to the diagonal embedding $d_{S^{\prime}}^{(n)}: G_{n, S^{\prime}} \rightarrow G_{n, S^{\prime}}^{n-1}$. Now, for every $n \geq 3$, let

$$
c^{(n)}: G_{n}^{n-1} \rightarrow G_{n}^{n-2},\left(x_{1}, \ldots, x_{n-1}\right) \mapsto\left(x_{1} x_{n-1}^{-1}, \ldots, x_{n-2} x_{n-1}^{-1}\right),
$$


and let $c^{(2)}: G_{2} \rightarrow S$ be the structural morphism of $G_{2}$. Then $c^{(n)}$ is a morphism of commutative $S$-group schemes and there exists a canonical exact sequence of commutative $S$-group schemes

$$
0 \rightarrow G_{n} \stackrel{d^{(n)}}{\longrightarrow} G_{n}^{n-1} \stackrel{c^{(n)}}{\longrightarrow} G_{n}^{n-2} \rightarrow 0
$$

where $d^{(n)}$ is the diagonal $S$-morphism. Now recall $G(n) \in \mathrm{Ob} S_{\mathrm{fl}}^{\sim}$ (4.27). We define an isomorphism of $S^{\prime}$-group schemes $G(n)_{S^{\prime}} \stackrel{\sim}{\rightarrow} G_{n, S^{\prime}}^{n-2}$ by the commutativity of the diagram

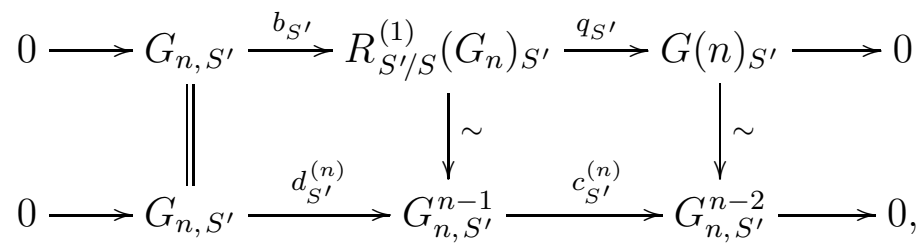

where the top (respectively, bottom) row is induced by (4.28) (respectively, (5.3)). We conclude that $G(2)=0$, whence $H^{1}\left(\Gamma_{2}^{\bullet}(r, G)\right)=0$ for every $r \geq 1$ (4.34). Thus the following statement is immediate from Theorem 4.2 and Corollary 4.3 using (4.23) and (5.2) for $n=2$.

Theorem 5.1. Let $f: S^{\prime} \rightarrow S$ be a quadratic Galois covering of locally noetherian schemes with Galois group $\Delta$ and let $G$ be a smooth, commutative and quasi-projective $S$-group scheme with connected fibers. Assume that, for every point $s \in S$ such that char $k(s)=2, G_{k(s)}$ is a semiabelian $k(s)$-variety. Then, for every $r \geq 1$, there exist canonical exact sequences of 2-torsion abelian groups

$$
0 \rightarrow \operatorname{Ker}\left(\operatorname{Res}_{G}^{(r-1)} / 2\right) \rightarrow \operatorname{Ker} j_{G_{2}, \mathrm{fl}}^{(r)} \rightarrow \operatorname{Ker}_{\operatorname{Res}_{G}^{(r)}}^{(r)} \rightarrow H^{1}\left(C_{/ 2}^{\bullet}(r-1, G)\right) \rightarrow 0
$$

and

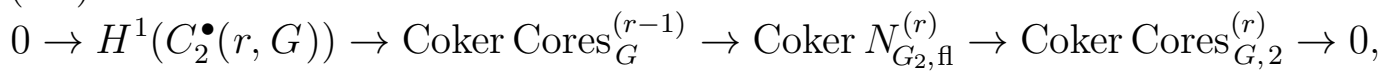

where the complexes $C_{/ 2}^{\bullet}(r-1, G)$ and $C_{2}^{\bullet}(r, G)$ are given by (4.13) and (4.15), respectively. In particular, if $r=1$, then (5.4) induces an exact sequence of 
2-torsion abelian groups

$$
0 \rightarrow \frac{G(S) \cap G\left(S^{\prime}\right)^{2}}{G(S)^{2}} \rightarrow H^{1}\left(\Delta, G\left(S^{\prime}\right)_{2}\right) \rightarrow \operatorname{Ker} \operatorname{Res}_{G}^{(1)} \rightarrow \frac{\Psi_{N}(2, G)}{G(S) G\left(S^{\prime}\right)^{2}} \rightarrow 0,
$$

where $\Psi_{N}(2, G)=\left\{x \in G\left(S^{\prime}\right): N_{S^{\prime} / S}(x) \in G(S)^{2}\right\}$.

The above theorem yields, in particular, the following "lower bound" for the relative cohomological Brauer group of a quadratic Galois covering of locally noetherian schemes in terms of their Picard groups.

Corollary 5.2. Let $f: S^{\prime} \rightarrow S$ be a quadratic Galois covering of locally noetherian schemes. Then there exists a canonical surjection of 2-torsion abelian groups

$$
\operatorname{Br}^{\prime}\left(S^{\prime} / S\right) \rightarrow \frac{\operatorname{Ker}\left[\operatorname{Pic} S^{\prime} / 2 \stackrel{\text { Cores }_{S^{\prime} / S}}{\longrightarrow} \operatorname{Pic} S / 2\right]}{\operatorname{Im}\left[\operatorname{Pic} S / 2 \stackrel{\operatorname{Res}_{S^{\prime} / S}^{\longrightarrow}}{\longrightarrow} \operatorname{Pic} S^{\prime} / 2\right]},
$$

where $\operatorname{Br}^{\prime}\left(S^{\prime} / S\right)=\operatorname{Ker}\left[\operatorname{Res}_{S^{\prime} / S}: \mathrm{Br}^{\prime} S \rightarrow \mathrm{Br}^{\prime} S^{\prime}\right]$ is the relative cohomological Brauer group of $S^{\prime}$ over $S$.

Proof. Set $r=2$ and $G=\mathbb{G}_{m, S}$ in (5.4) and note that, by (4.13),

$$
H^{1}\left(C_{/ 2}^{\bullet}\left(1, \mathbb{G}_{m, S}\right)\right)=\frac{\operatorname{Ker}\left[\operatorname{Pic} S^{\prime} / 2 \stackrel{\text { Cores }_{S^{\prime} / S}}{\longrightarrow} \operatorname{Pic} S / 2\right]}{\operatorname{Im}\left[\operatorname{Pic} S / 2 \stackrel{\operatorname{Res}_{S^{\prime} / S}^{\longrightarrow}}{\longrightarrow} \operatorname{Pic} S^{\prime} / 2\right]} .
$$

Remark 5.3. Assume that $S$ is a noetherian scheme which admits an ample invertible sheaf, and similarly for $S^{\prime}$. Then $\left(\operatorname{Br} S^{\prime}\right)_{2}=(\operatorname{Br} S)_{2}$ and similarly for $S^{\prime}$ by (2.2). Thus, setting $r=2$ and $G=\mathbb{G}_{m, S}$ in (5.5), we recover the Knus-Parimala-Srinivas injection [19, 23]:

$$
\frac{\operatorname{Ker}\left[\left(\operatorname{Br} S^{\prime}\right)_{2} \stackrel{\operatorname{Cores}_{S^{\prime} / S}}{\longrightarrow}(\operatorname{Br} S)_{2}\right]}{\operatorname{Im}\left[(\operatorname{Br} S)_{2} \stackrel{\operatorname{Res}_{S^{\prime} / S}^{\longrightarrow}}{\longrightarrow}\left(\operatorname{Br} S^{\prime}\right)_{2}\right]} \hookrightarrow \frac{\operatorname{Pic} S}{\operatorname{Cores}_{S^{\prime} / S}\left(\operatorname{Pic} S^{\prime}\right)}
$$




\section{Arithmetical applications}

Recall that a global field is either a number field, i.e., a finite extension of $\mathbb{Q}$, or a global function field, i.e., the function field of a smooth, projective and irreducible algebraic curve over a finite field.

Let $K / F$ be a quadratic Galois extension of global fields with Galois group $\Delta$ and let $\Sigma$ be a nonempty finite set of primes of $F$ containing the archimedean primes and the non-archimedean primes that ramify in $K$. Let $\Sigma_{K}$ be the set of primes of $K$ that lie above the primes in $\Sigma$, write $\mathcal{O}_{F, \Sigma}$ for the ring of $\Sigma$ integers of $F$ and let $\mathcal{O}_{K, \Sigma_{K}}$ be the ring of $\Sigma_{K}$-integers of $K$. If $S=\operatorname{Spec} \mathcal{O}_{F, \Sigma}$ and $S^{\prime}=\operatorname{Spec} \mathcal{O}_{K, \Sigma_{K}}$, then the canonical morphism $f: S^{\prime} \rightarrow S$ induced by the inclusion $\mathcal{O}_{F, \Sigma} \subset \mathcal{O}_{K, \Sigma_{K}}$ is a quadratic Galois covering. Now let $G_{F}$ be a smooth, commutative and connected $F$-group scheme of finite type which admits a Néron-Raynaud model over $S$ with semiabelian reduction at every point $s \in S$ such that char $k(s)=2$. If $G$ denotes the identity component of the indicated model, then the pair $(f, G)$ satisfies the hypotheses of Theorem 5.1. In this section we will consider the following specific choices of $G_{F}$ :

(1) $G_{F}$ is an invertible $F$-torus, i.e., $G_{F}$ is isomorphic to a direct factor of a finite direct product of $F$-tori of the form $R_{L / F}\left(\mathbb{G}_{m, L}\right)$, where $L / F$ is a finite separable extension of $F$, with multiplicative (i.e., toric) reduction at every point $s \in S$ such that $\operatorname{char} k(s)=2$.

(2) $G_{F}$ is an abelian variety over $F$ with abelian (i.e., good) reduction over $S$, i.e., $G$ is an abelian scheme over $S$.

Remark 6.1. In Case (2) above, $G(S)=G(F)$ and $G\left(S^{\prime}\right)=G(K)$ since $G$ and $G_{S^{\prime}}$ are Néron models of $G_{F}$ and $G_{K}=G_{F} \times_{F} K$, respectively [6, $\S 1.2$, Proposition 8, p. 15].

If $G_{F}$ is a smooth, commutative and connected $F$-group scheme of finite type which admits a Néron-Raynaud model over $S$ with identity component $G$, the Néron-Raynaud $\Sigma$-class group of $G_{F}$, introduced in [11, §3], is the quotient

$$
C_{G_{F}, F, \Sigma}=G\left(\mathbb{A}_{S}\right) / G(F) G\left(\mathbb{A}_{S}(S)\right),
$$

where $\mathbb{A}_{S}$ (respectively, $\mathbb{A}_{S}(S)$ ) is the ring of adeles (respectively, integral adeles) of $S$. The above group is known to be finite if $G_{F}$ is affine [8, $\$ 1.3$ ] (and thus for every $G_{F}$ in Case (1) above) and coincides with the $\Sigma$-ideal class group of $F$ when $G_{F}=\mathbb{G}_{m, F}$. In general, (6.1) is a quotient of $\bigoplus_{s \in S} \Phi_{s}\left(G_{F}\right)(k(s))$, where $\Phi_{s}\left(G_{F}\right)$ is the $k(s)$-group scheme of connected components of the special 
fiber of the Néron-Raynaud model of $G_{F_{v}}:=G_{F} \times_{F} F_{v}$, where $v \notin \Sigma$ is the prime of $F$ that corresponds to $s \in S$ [11, Theorem 3.2]. Consequently, (6.1) is also finite if $G_{F}$ is an abelian variety over $F$. Further, (6.1) is trivial in Case (2) above. The capitulation problem for $C_{G_{F}, F, \Sigma}$ (relative to $K / F$ ) is the problem of describing the kernel of the map $j_{G_{F}, K / F, \Sigma}: C_{G_{F}, F, \Sigma} \rightarrow C_{G_{K}, K, \Sigma_{K}}$ induced by $f$. This problem has a long history in the classical case $G_{F}=\mathbb{G}_{m, F}$ when $F$ is a number field (see, e.g., [24]) and was discussed in [10, §4] for arbitrary finite Galois extension of global fields $K / F$ and arbitrary $F$-tori $G_{F}$ with multiplicative reduction over $S$.

Next, the $\Sigma$-Tate-Shafarevich group of $G_{F}$ is the group

$$
\amalg_{\Sigma}^{1}(F, G)=\operatorname{Ker}\left[H^{1}(F, G) \rightarrow \prod_{v \notin \Sigma} H^{1}\left(F_{v}, G\right)\right],
$$

where $H^{1}(F, G)=H^{1}\left(F, G_{F}\right)$ and $H^{1}\left(F_{v}, G\right)=H^{1}\left(F_{v}, G_{F_{v}}\right)$ are Galois cohomology groups. For every prime $v$ of $F$, fix a prime $v^{\prime}$ of $K$ lying above $v$ and set $\Delta_{v^{\prime}}=\operatorname{Gal}\left(K_{v^{\prime}} / F_{v}\right)$. Every prime $w$ of $K$ lying above $v$ has the form $\sigma v^{\prime}$ for some $\sigma \in \Delta$ and $\sigma$ induces an isomorphism of abelian groups $H^{1}\left(K_{w}, G\right) \stackrel{\sim}{\rightarrow} H^{1}\left(K_{v^{\prime}}, G\right)$. It follows that the $\Sigma_{K^{-}}$Tate-Shafarevich group of $G_{K}:=G_{F} \times{ }_{F} K$ equals

$$
\amalg_{\Sigma}^{1}(K, G):=\operatorname{Ker}\left[H^{1}(K, G) \rightarrow \prod_{v \notin \Sigma} H^{1}\left(K_{v^{\prime}}, G\right)\right] .
$$

The capitulation problem for $\amalg_{\Sigma}^{1}(F, G)$ (relative to $K / F$ ) is the problem of describing the kernel of the map $\amalg_{\Sigma}^{1}\left(\operatorname{Res}_{G, K / F}^{(1)}\right): \amalg_{\Sigma}^{1}(F, G) \rightarrow \amalg_{\Sigma}^{1}(K, G)$ induced by $\operatorname{Res}_{G, K / F}^{(1)}: H^{1}(F, G) \rightarrow H^{1}(K, G)$. By the inflation-restriction exact sequence in Galois cohomology [3, Proposition 4, p. 100], the exact and commutative diagram of abelian groups

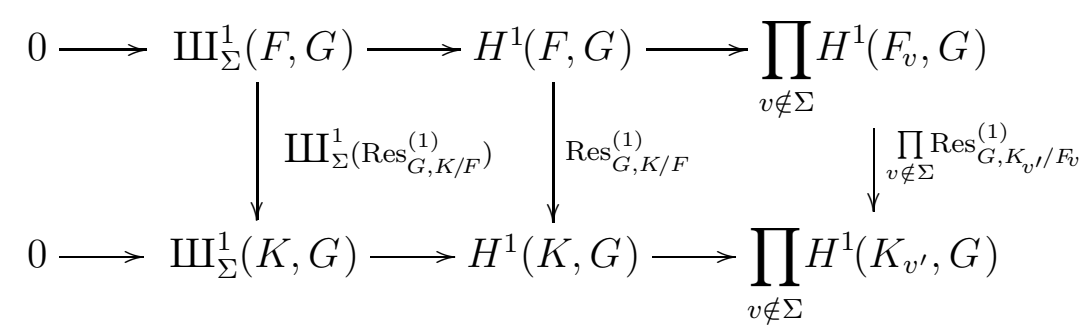


yields the equality

$$
\text { Ker } \amalg_{\Sigma}^{1}\left(\operatorname{Res}_{G, K / F}^{(1)}\right)=\amalg_{\Sigma}^{1}(\Delta, G(K)),
$$

where 5

$$
\amalg_{\Sigma}^{1}(\Delta, G(K))=\operatorname{Ker}\left[H^{1}(\Delta, G(K)) \rightarrow \prod_{v \notin \Sigma} H^{1}\left(\Delta_{v^{\prime}}, G\left(K_{v^{\prime}}\right)\right)\right] .
$$

Now, by [11, (3.10)], there exists a canonical exact sequence of abelian groups

$$
0 \rightarrow C_{G_{F}, F, \Sigma} \rightarrow H^{1}\left(S_{\text {ét }}, G\right) \rightarrow \amalg_{\Sigma}^{1}(F, G) \rightarrow 0 .
$$

A similar exact sequence exists over $K$ if $G \times{ }_{S} S^{\prime}$ agrees with the identity component of the Néron-Raynaud model of $G_{K}$ over $S^{\prime}$, which is the case here since $S^{\prime}$ is étale over $S$ (see [6, $\S 7.2$, Theorem 1 (ii), p. 176] and [9, $\mathrm{VI}_{\mathrm{B}}$, Proposition 3.3]). Thus there exists a canonical exact and commutative diagram of abelian groups

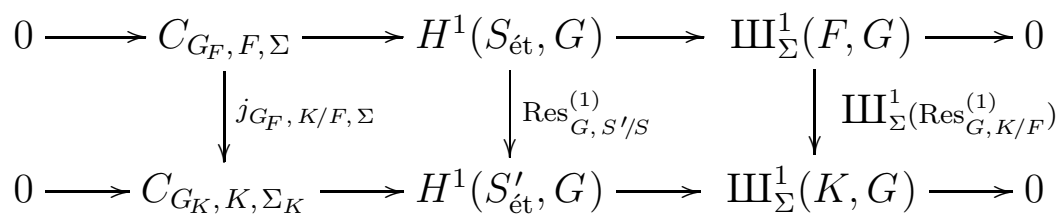

By (6.2), the preceding diagram induces an exact sequence of abelian groups

$$
0 \rightarrow \operatorname{Ker} j_{G_{F}, K / F, \Sigma} \rightarrow \operatorname{Ker} \operatorname{Res}_{G, S^{\prime} / S}^{(1)} \rightarrow \amalg_{\Sigma}^{1}(\Delta, G(K)) .
$$

In Case (1) above, i.e., $G_{F}$ is an invertible $F$-torus, the group $\amalg_{\Sigma}^{1}(\Delta, G(K))$ (which is isomorphic to a subgroup of $H^{1}(F, G)$ via the inflation map) is trivial since $H^{1}(F, G)$ is trivial by Hilbert's Theorem 90 [10, Lemma 4.8(a)]. In this case (6.5) reduces to a canonical isomorphism of abelian groups

$$
\operatorname{Ker} j_{G_{F}, K / F, \Sigma}=\operatorname{Ker} \operatorname{Res}_{G, S^{\prime} / S}^{(1)} \text {. }
$$

In Case (2), i.e., $G$ is an abelian scheme over $S$, the groups $C_{G_{F}, F, \Sigma}$ and $C_{G_{K}, K, \Sigma_{K}}$ are trivial (as noted previously) and (6.4) over $F$ and over $K$ yield

\footnotetext{
${ }^{5}$ Here we write $G(K)$ for $G_{K}(K)$ and $G\left(K_{v^{\prime}}\right)$ for $G_{K_{v^{\prime}}}\left(K_{v^{\prime}}\right)$.
} 
isomorphisms $H^{1}\left(S_{\text {ét }}, G\right)=\amalg_{\Sigma}^{1}(F, G)$ and $H^{1}\left(S_{\text {ét }}^{\prime}, G\right)=\amalg_{\Sigma}^{1}(K, G)$. In this case (6.5) reduces to a canonical isomorphism of abelian groups

$$
\amalg_{\Sigma}^{1}(\Delta, G(K))=\operatorname{Ker} \operatorname{Res}_{G, S^{\prime} / S}^{(1)} .
$$

Theorem 5.1 (or, more precisely, sequence (5.6)) and Remark 6.1 yield the following statement.

Theorem 6.2. Let $K / F$ be a quadratic Galois extension of global fields with Galois group $\Delta$ and let $\Sigma$ be a nonempty finite set of primes of $F$ containing the archimedean primes and the non-archimedean primes that ramify in $K$. Set $S=\operatorname{Spec} \mathcal{O}_{F, \Sigma}$ and $S^{\prime}=\mathcal{O}_{K, \Sigma_{K}}$.

(i) If $T$ is an invertible torus over $F$ with multiplicative reduction at every point $s \in S$ such that char $k(s)=2$, then there exists a canonical exact sequence of 2-torsion abelian groups

$\frac{\mathcal{T}^{0}(S) \cap \mathcal{T}^{0}\left(S^{\prime}\right)^{2}}{\mathcal{T}^{0}(S)^{2}} \hookrightarrow H^{1}\left(\Delta, \mathcal{T}^{0}\left(S^{\prime}\right)_{2}\right) \rightarrow \operatorname{Ker} j_{T, K / F, \Sigma} \rightarrow \frac{\Psi_{N}\left(2, \mathcal{T}^{0}\right)}{\mathcal{T}^{0}(S) \mathcal{T}^{0}\left(S^{\prime}\right)^{2}}$,

where $\mathcal{T}^{0}$ is the identity component of the Néron-Raynaud model of $T$ over $S, j_{T, K / F, \Sigma}: C_{T, F, \Sigma} \rightarrow C_{T, K, \Sigma_{K}}$ is the canonical capitulation map for Néron-Raynaud $\Sigma$-class groups of $T$ relative to the extension $K / F$ and $\Psi_{N}\left(2, \mathcal{T}^{0}\right)=\left\{x \in \mathcal{T}^{0}\left(S^{\prime}\right): N_{S^{\prime} / S}(x) \in \mathcal{T}^{0}(S)^{2}\right\}$.

(ii) If $\mathcal{A}$ is an abelian scheme over $S, A=\mathcal{A}_{F}$ and $\amalg_{\Sigma}^{1}(\Delta, A(K))$ is the group (6.3), then there exists a canonical exact sequence of 2-torsion abelian groups

$$
\begin{aligned}
& \frac{A(F) \cap 2 A(K)}{2 A(F)} \hookrightarrow H^{1}\left(\Delta, A(K)_{2}\right) \rightarrow \amalg_{\Sigma}^{1}(\Delta, A(K)) \rightarrow \frac{\Psi_{N}(2, A)}{(A(F)+2 A(K))}, \\
& \text { where } \Psi_{N}(2, A)=\left\{P \in A(K): N_{K / F}(P) \in 2 A(F)\right\} .
\end{aligned}
$$

Setting $T=\mathbb{G}_{m, F}$ in part (i) of the theorem, we obtain the following statement about the classical capitulation kernel $\operatorname{Ker} j_{K / F, \Sigma}$.

Corollary 6.3. Let $K / F$ be a quadratic Galois extension of global fields and let $\Sigma$ be a nonempty finite set of primes of $F$ containing the archimedean primes and the non-archimedean primes that ramify in $K$. Then there exists a canonical exact sequence of finite 2-torsion abelian groups

$$
0 \rightarrow \frac{\mathcal{O}_{F, \Sigma}^{*} \cap\left(\mathcal{O}_{K, \Sigma}^{*}\right)^{2}}{\left(\mathcal{O}_{F, \Sigma}^{*}\right)^{2}} \rightarrow\{ \pm 1\} \rightarrow \operatorname{Ker} j_{K / F, \Sigma} \rightarrow \frac{\Psi_{N}(K / F, \Sigma)}{\mathcal{O}_{F, \Sigma}^{*}\left(\mathcal{O}_{K, \Sigma}^{*}\right)^{2}} \rightarrow 0
$$


where

$$
\Psi_{N}(K / F, \Sigma)=\left\{u \in \mathcal{O}_{K, \Sigma}^{*}: N_{K / F}(u) \in\left(\mathcal{O}_{F, \Sigma}^{*}\right)^{2}\right\}
$$

Remark 6.4. In those cases where the group $\mathcal{O}_{F, \Sigma}^{*} \cap\left(\mathcal{O}_{K, \Sigma}^{*}\right)^{2} /\left(\mathcal{O}_{F, \Sigma}^{*}\right)^{2}$ has order 2 , the sequence of the corollary yields an isomorphism

$$
\operatorname{Ker} j_{K / F, \Sigma} \simeq \frac{\left\{u \in \mathcal{O}_{K, \Sigma}^{*}: N_{K / F}(u) \in\left(\mathcal{O}_{F, \Sigma}^{*}\right)^{2}\right\}}{\mathcal{O}_{F, \Sigma}^{*}\left(\mathcal{O}_{K, \Sigma}^{*}\right)^{2}}
$$

which differs from the standard isomorphism

$$
\operatorname{Ker} j_{K / F, \Sigma} \simeq \frac{\left\{u \in \mathcal{O}_{K, \Sigma}^{*}: N_{K / F}(u)=1\right\}}{(1-\tau) \mathcal{O}_{K, \Sigma}^{*}}
$$

noted in the Introduction (above $\tau$ is the nontrivial element of $\Delta$ ). For a variant of Corollary 6.3 which applies to arbitrary finite Galois extensions of global fields $K / F$, see [12, Corollary 4.2].

\section{REFERENCES}

[1] Anantharaman, S.: Schémas en groupes, espaces homogènes et espaces algébriques sur une base de dimension 1. Mem. Soc. Math. France, 33 (1973), 5-79.

[2] Atiyah, M. and MacDonald, I.: Introduction to commutative algebra, Addison-Wesley Publishing Company, Inc., Reading, MA (1969).

[3] Atiyah, M. and Wall, C.T.C.: Cohomology of groups, in: Algebraic Number Theory

(J.W.S. Cassels and A. Fröhlich, Eds.), 94-115, Academic Press, London, 1967.

[4] Beyl, R.: The connecting morphism in the kernel-cokernel sequence. Arch. der Math. 32 (1979), no. 4, 305-308.

[5] Bertapelle, A. and González-Avilés, C.D. The Greenberg functor revisited. arXiv:1311.0051v4.

[6] Bosch, S., Lütkebohmert, W. and Raynaud, M. Néron Models. Springer Verlag, Berlin 1989.

[7] Conrad, B., Gabber, O. and Prasad, G.: Pseudo-reductive groups. Second Ed. New Math. Monograps 26, Cambridge U. Press, 2015.

[8] Conrad, B.: Finiteness theorems for algebraic groups over function fields. Comp. Math. 148 (2012), no. 2, 555-639.

[9] Demazure, M. and Grothendieck, A. (Eds.): Schémas en groupes. Séminaire de Géométrie Algébrique du Bois Marie 1962-64 (SGA 3). Augmented and corrected 2008-2011 re-edition of the original by P.Gille and P.Polo. Available at http://www.math.jussieu.fr/ polo/SGA3. Reviewed at http://www.jmilne.org/math/xnotes/SGA3r.pdf. 
[10] González-Avilés, C.D. On Néron-Raynaud class groups of tori and the capitulation problem. J. reine angew. Math. 648 (2010), 149-182.

[11] González-Avilés, C.D. On Néron class groups of abelian varieties. J. reine angew. Math. 664 (2012), 71-91.

[12] González-Avilés, C.D. Čech cohomology and the Capitulation kernel. Funct. Approx. Comment. Math. doi: 10.7169/facm/1758.

[13] Grothendieck, A.: Revêtements Étales et groupe fondamental. Séminaire de Géométrie Algébrique du Bois Marie 1960-61 (SGA 1). Lect. Notes in Math. 224, Springer-Verlag 1973.

[14] Grothendieck, A. and Dieudonné, J.: Éléments de géométrie algébrique. Publ. Math.

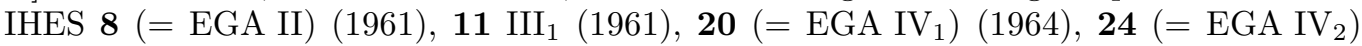
(1965), 32 (=EGA IV 4 ) (1967).

[15] Grothendieck, A. and Dieudonné, J.: Éléments de géométrie algébrique I. Le langage des schémas. Grund. der Math. Wiss. 166 (1971).

[16] Grothendieck, A. et al.: Théorie des Topos et Cohomologie Étale des Schémas. Séminaire de Géométrie Algébrique du Bois Marie 1963-64 (SGA 4). Lect. Notes in Math. 270, 305 and 370, Springer-Verlag 1973.

[17] Grothendieck, A.: Le groupe de Brauer I-III. In: Dix Exposés sur la cohomologie des schémas, North Holland, Amsterdam, 1968, 46-188.

[18] de Jong, J. A result of Gabber (unpublished). Available at wWw. math.columbia.edu/ dejong/papers/2-gabber.pdf.

[19] Knus, M.-A., Parimala, R. and Srinivas, V. Azumaya algebras with involutions. J. Algebra 130 (1990), no. 1, 65-82.

[20] Milne, J.S.: Étale cohomology. Princeton University Press, Princeton, 1980.

[21] Moret-Bailly, L.: Answer to https://mathoverflow.net/questions/285419.

[22] Oesterlé, J.: Nombres de Tamagawa et groupes unipotents en caractérisque p. Invent. Math. 78 (1984), 13-88.

[23] Parimala, R. and Srinivas, V. Analogues of the Brauer group for algebras with involution. Duke Math. J. 66 (1992), no. 2, 207-237.

[24] Scholz, A. and Taussky, O. Die Hauptideale der kubischen Klassenkörper imaginär-quadratische Zahlkörper: ihre rechnerische Bestimmung und ihr Einfluß auf den Klassenkörperturm. J. reine angew. Math. 171 (1934), 19-41.

[25] The Stacks Project. http://stacks.math.columbia.edu

[26] Tamme, G.: Introduction to Étale Cohomology. Translated from the German by Manfred Kolster. Universitext. Springer-Verlag, Berlin, 1994.

Departamento de Matemáticas, Universidad de La Serena, La Serena, Chile E-mail address: cgonzalez@userena.cl 